PROCEEDINGS OF THE

AMERICAN MATHEMATICAL SOCIETY

Volume 46, Number 2, November 1974

\title{
NORMAL MOORE SPACES IN THE CONSTRUCTIBLE UNIVERSE
}

\author{
WILLIAM FLEISSNER 1
}

ABSTRACT. Assuming the axiom of constructibility, points in closed discrete subspaces of certain normal spaces can be simultaneously separated. This is a partial result towards the normal Moore space conjecture.

The normal Moore space conjecture states that every normal Moore space is metrizable. This is known to be not provable from the usual axioms of set theory, since Silver [4] shows that Martin's axiom with the negation of $\mathrm{CH}$ implies the existence of a separable nonmetrizable normal Moore space. In this paper we consider the situation under Gödel's axiom of constructibility $(V=L)$.

Bing [1] shows that a normal Moore space is metrizable iff it is collectionwise normal. Moore spaces have character $\boldsymbol{k}_{0}$ (i.e. are first countable). The following is then a partial result towards proving the normal Moore space conjecture in $L$.

Theorem $(V=L)$. If $X$ is a normal Hausdorff space of character $\leq \boldsymbol{x}_{1}$, then $X$ is collectionwise Hausdorff.

Definition. A space is collectionwise Hausdorff $(\mathrm{CWH})$ iff every closed discrete set of points can be simultaneously separated by disjoint open sets. Let $\mathrm{CWH}(\kappa)$ be $\mathrm{CWH}$ restricted to sets of cardinality $\leq \kappa$.

Remarks. 1. The Theorem shows consistent Bing's conjecture [2] that normal Moore spaces be CWH. It shows in fact that in a normal character $\boldsymbol{x}_{1}$ space, a discrete collection of countable closed sets can be simultaneously separated by the device of shrinking each closed set to a point, which then is of character $x_{1}$. 1973.

Received by the editors August 2, 1973 and, in revised form, September 30, $54 \mathrm{E} 30$.

AMS (MOS) subject classifications (1970). Primary 02K05, 04A20, 54D15,

Key words and phrases. Normal Moore spaces, combinatorial principles in $L$, constructible universe, cardinal functions in topology.

1 While working on this paper, the author was a National Science Foundation Graduate Fellow.

Copyright $\odot 1974$, American Mathematical Society 
$2^{x_{1}}$ which is not CWH.

3. Tall [4] shows the consistency of $\mathrm{CWH}(\kappa)$ for all $\kappa<x_{\omega_{1}}$, using forcing rather than $L$.

4. The author has constructed a normal CWH, not collectionwise normal space of character $c$.

5. The Theorem implies that every locally compact normal Moore space is metrizable.

6. Kunen asks whether $V=L$ can be weakened to $\mathrm{GCH}$.

Let us fix $X$, a normal Hausdorff space of character $\aleph_{1}$. We will prove $\mathrm{CWH}(\kappa)$ for all $\kappa$ by induction on $\kappa \cdot \mathrm{CWH}\left(\boldsymbol{\aleph}_{0}\right)$ is a consequence of the regularity of $X$. The same argument, using normality, yields:

A countable, closed, discrete collection of closed sets can be simultaneously separated by disjoint open sets.

For singular $\kappa$ of cofinality $\boldsymbol{x}_{0}\left(\operatorname{cf}(\kappa)=\boldsymbol{x}_{0}\right), \mathrm{CWH}(\kappa)$ follows from $(*)$ and the induction hypothesis. Henceforth, we will implicitly assume $\mathrm{CWH}(\lambda)$ for $\lambda<\kappa$ :

Definition. A set $C \subset \kappa$ is $c u b$ (closed, unbounded) iff $C$ is unbounded and closed in the order topology. A set $A \subset \kappa$ is stationary (alternatively, Mahlo) iff $A \cap C \not \varnothing$ for all cub $C$. It is easy to show that the intersection of 2 (in fact, of $<\operatorname{cf}(\kappa)$ ) cub sets is cub, hence the intersection of a cub set and a stationary set is stationary.

Cub sets can also be characterized as follows. For $B \subset \kappa$, define $B^{*}(\alpha)$ to be the greatest $\beta \in B$ such that $\beta \leq \alpha$, if such a $\beta$ exists. Define $\alpha \sim_{B} \beta$ iff $\alpha \cap B=\beta \cap B$ so that $B^{*}(\alpha)=B^{*}(\beta)$ if defined. Then $B$ is closed iff $B^{*}(\alpha)$ is always defined, and $B$ is unbounded iff each $\sim_{B}$ equivalence class has cardinality $<\kappa$.

To simplify notation, assume that $\kappa$ is an arbitrary closed discrete subset of $X$, so that if we separate $\kappa$ we may conclude CWH( $\kappa)$. For $f: \kappa \rightarrow \omega_{1}$, let $f^{\#}(\alpha)$ be the $f(\alpha)$ th basis set at the point $\alpha$. Then any separation $U, V$ of $H, K \subset \kappa$ has a refinement that is coded by an $f: \kappa \rightarrow \omega_{1}$. Let $W_{a}^{f}=$ $\bigcup\left\{f^{\#}(\beta): \beta<\alpha\right\}$.

We will go through $\kappa$ assigning points to $H$ and $K$, considering initial segments $W_{a}^{f}$ of potential separations, and destroying them if we can. For regular $\kappa$ we will assume that $\kappa \subset X$ witnesses $7 \mathrm{CWH}(\kappa)$, and conclude from Lemma 1 that each $f$ can be destroyed on a stationary set. Then using the combinatorial principle (Lemma 2 ) to tell us which $f$ to consider at stage $a$, 
we will, in Lemma 3, define $H$ and $K$ that cannot be separated-contradicting the normality of $X$, thus establishing $\mathrm{CWH}(\kappa)$.

Lemma 1. Either $A_{f}=\left\{\alpha<\kappa: \overline{W_{\alpha}^{\prime}} \cap \kappa \neq \alpha\right\}$ is stationary for all $f: \kappa \rightarrow$ $\omega_{1}$ or the points in $\kappa$ can be simultaneously separated.

Proof. If $A_{f}$ is not stationary, there is a cub $C$ such that $a \in C$ implies $W_{\alpha}^{f} \cap \kappa=\alpha$. This means that there is a $g$ so that $g^{\#}(\alpha) \cap f^{\#}(\beta)=\varnothing$ for all $\beta<C^{*}(\alpha)$. By the induction hypothesis there is an $b$ so that $\alpha \sim_{C} \beta$ implies $b^{\#}(\alpha) \cap b^{\#}(\beta)=\varnothing$. Then $f^{\#}(\alpha) \cap g^{\#}(\alpha) \cap b^{\#}(\alpha)$ separates the points of $\kappa$.

Define a stationary system to be a function $A:{ }^{\kappa} \lambda \rightarrow P(\kappa)$ such that $\kappa \geq \lambda, A_{f}$ is stationary for every $f$, and

$$
f \vdash \alpha=g\left\lceil\alpha \rightarrow A_{f} \cap(\alpha+1)=A_{g} \cap(\alpha+1) .\right.
$$

By (**), $\alpha \in A_{f}$ is meaningful whenever $\operatorname{dom}(f) \supseteq \alpha$. If $\kappa$ cannot be separated, then the $A$ defined in Lemma 1 is a stationary system.

Lemma $2(V=L)$. If $A$ is a stationary system and $k$ is regular, then there is a $\Phi: S \subset \kappa \rightarrow \bigcup\left\{{ }^{a} \alpha, a<\kappa\right\}$ such that:

(i) $\Phi(\alpha): \alpha \rightarrow \alpha$.

(ii) For all $f \in{ }^{\kappa} \lambda, B_{f}=\{\alpha: \Phi(\alpha)=f \uparrow \alpha\}$ is a stationary subset of $A_{f^{*}}$

If $A_{f}=A$ for all $f \epsilon^{\kappa} \lambda$, then Lemma 2 is Jensen's $\vartheta(A)$ [3]. We follow Jensen's proof, which uses the ideas of elementary submodel, transitive collapse, and the condensation lemma for $L$. The idea of the proof is as follows. $L$ has a very nice well ordering $<$. We define $\Phi(\alpha)=f \epsilon^{\alpha_{\alpha}}$ where $\langle f, C\rangle$ is the <-first such that $\alpha \in A_{f}, C$ is cub $\subset \alpha$, and $B, \cap C=\varnothing$. If there were a counterexample to the lemma, there would be a <-least one $\left\langle f_{0}, C_{0}\right\rangle$. With ordinary set theory it is easy to show that for a cub of $\alpha$ 's, $f_{0} \uparrow a \in{ }^{a} a$ and $C_{0} \cap a$ is cub in $\alpha$. Moreover, assuming $V=L$, we get $\left\langle f_{0} \uparrow a, C_{0} \uparrow a\right\rangle$ is the <-first counterexample for a cub $C^{\prime}$ of $\alpha$ 's. But then $B_{f_{0}}=C^{\prime} \cap A_{f_{0}}$ is stationary, a contradiction.

Proof. Define $\Phi(\alpha)$ by induction on $\alpha . \Phi(\alpha)=f$ where $\langle f, C\rangle$ is the <first pair (if there is one; if there is none, leave $\Phi(a)$ undefined) such that 1. $f \epsilon^{\alpha} \alpha, C$ is cub in $\alpha$.

2. If $\langle g, D\rangle\langle\langle f, C\rangle$ satisfies 1 , then there is $\beta \subset \alpha$ such that $\Phi(\beta)=$ $g \mid \beta$ and $\beta \in D \cap A_{g^{*}}$

3. $\alpha \in A_{f}$.

We claim $\Phi$ works. If not, let $\left\langle f_{0}, C_{0}\right\rangle$ be the <-first counterexample. 
Define a continuous monotone increasing sequence $M_{\alpha}, \alpha<\kappa$, of elementary submodels of $L_{\kappa^{++}}$containing $A$ and $\left\langle f_{0}, C_{0}\right\rangle$, such that $M_{\alpha} \cap \kappa \in$ OR and card $M_{a}<\kappa$. Let $\pi_{a}$ be the transitive collapse of $M_{\alpha}$, and let $\delta(\alpha)$ be $\pi_{\alpha}(\kappa)$. Then

(a) $C^{\prime}=\{\delta(\alpha): \alpha<\kappa\}$ is cub in $\kappa$,

(b) for $f \in \epsilon^{\kappa} \lambda \cap M_{a}, \pi_{a}(f)=f \uparrow \delta(a)$,

(c) for $C \in P(\kappa) \cap M_{\alpha}, \pi_{\alpha}(c)=\delta(\alpha) \cap C$.

Thus for $\alpha \in P^{\prime},\left\langle f_{0} \cap \alpha, C_{0} \cap \alpha\right\rangle$ is <-least such that 1 and 2 hold. If $\alpha \in C^{\prime} \cap A_{f_{0}}, 3$ holds as well, so $\Phi(\alpha)=f_{0} \uparrow \alpha$, a contradiction.

Lemma $3(V=L)$. CWH $(\lambda)$ for all $\lambda<\kappa$ implies $\mathrm{CWH}(\kappa)$ for regular $\kappa$.

Proof. Define $H, K, U_{a}^{f}, V_{a}^{f}$ by induction on $\alpha$.

$U_{a}^{f}=\bigcup\left\{f^{\#}(\beta): \beta<\alpha, \beta \in H\right\}, \quad V_{a}^{f}=\bigcup\left\{f^{\#}(\beta): \beta<\alpha, \beta \in K\right\}$.

Then $W_{\alpha}^{f}=U_{\alpha}^{f} \cup V_{\alpha}^{f}$. Suppose $\beta$ has been placed in $H$ or $K$ for all $\beta<\alpha$. Put $\alpha$ in $H$ unless $\Phi(\alpha)$ is defined, in which case $\overline{W_{\alpha}^{\Phi(\alpha)}} \cap \kappa-\alpha \neq \varnothing$ and has a least element $\nu(\alpha)$. If $\nu(\alpha) \in \overline{U_{a}^{\Phi(\alpha)}}$, place $\nu(\alpha)$ in $K$; place it in $H$ otherwise. Put $\beta \in \nu(\alpha)-\alpha$ in $H$. By the regularity of $\kappa,\{\alpha: \beta<\alpha \rightarrow \nu(\beta)$ is not defined or $\nu(\beta)<\alpha\}$ is a cub $C^{\prime \prime}$.

If $H$ and $K$ could be separated, they could be separated by some $f^{\#}$. By Lemma 2, there is an $\alpha \in C^{\prime \prime}$ such that $\Phi(\alpha)=f \uparrow \alpha$. Then, if $\nu(\alpha) \in H$, it is a limit of points in the open sets of the cover $f^{\#}$ of $K$, and similarly with $H$ and $K$ interchanged. $H$ and $K$ are closed sets that cannot be separated. This contradiction follows from the assumption $7 \mathrm{CWH}(\kappa)$.

One of the ways that the regularity of $\kappa$ is vitally used in the above proof is in the proof of Lemma 3, where $C^{\prime \prime}$ shows that our arbitrary ordering of a subset of $X$ does not matter. We will use the following definitions to consider various orders on subsets of singular $\kappa$. Let $S \subset \kappa \subset X$, and let $\rho: S \rightarrow \kappa$ be one-to-one. The following depend on $S$ and $\rho$. Redefine $W_{a}^{f}$ as $\bigcup\left\{f^{\#}(\beta): \rho(\beta)<\alpha\right.$ and $\left.\beta \in S\right\}$, and let $D_{\alpha}^{f}=\overline{W_{\alpha}^{f}} \cap\{\beta: \rho(\beta) \geq \alpha$ and $\beta \in S\}$. Call $f$ thick wrt $S, \rho$ if there is an $\alpha$ such that card $D_{\alpha}^{f}>\alpha ; f$ is thin if not.

Lemma $4(\mathrm{GCH})$. For every $S, \rho$ there is an $f$ thin wrt $S, \rho$.

Proof. If not, we can repeat the argument of Lemma 3 to show $X$ is not normal. Define $H$ and $K$ by induction using $\rho$ and the Gödel pairing function $\alpha \mapsto\left\langle\alpha_{1}, \alpha_{2}\right\rangle$, which has the property that $\alpha<\max \left(\alpha_{1}^{+}, \alpha_{2}^{+}\right)$. At stage $\alpha$ consider (if meaningful) the $\alpha_{1}$ th function $f: a_{2} \rightarrow \omega_{1}$, and assign (if possible) 
a new point from $D_{a_{2}}^{f}$ to $H$ or $K$ to destroy $f$. Assign $\rho^{-1}\{\alpha\}$ arbitrarily if it exists and is not already assigned. No $g^{\#}$ separates $H$ and $K$, for at stage $\alpha$ only card $\alpha$ points have been assigned, so by thickness and GCH (Gödel: $V=L \rightarrow \mathrm{GCH}$ ), there was some $\alpha$ for which it was meaningful and possible to destroy $g$.

We need to choose $S, \rho$ carefully. Because $\kappa$ is singular, there is a set $C=\left\{c_{\alpha}: \alpha<\operatorname{cf}(\kappa)\right\}$ of cardinals $>\operatorname{cf}(\kappa)$ cub in $\kappa$ of order type $\mathrm{cf}(\kappa)$. Let

$$
B_{a}=\left\{\gamma: \gamma=\operatorname{cf}(\kappa) \cdot \delta+\alpha \text { for some } \delta<c_{\alpha}\right\} \text {. }
$$

Then $B_{\alpha}$ 's are disjoint subsets of $\kappa$ such that $B_{\alpha} \subset c_{\alpha}$, and card $B_{\alpha}=c_{\alpha}$.

Lemma $5(\mathrm{GCH})$. $\mathrm{CWH}(\lambda)$ for all $\lambda<\kappa$ implies $\mathrm{CWH}(\kappa)$ for $\kappa>\operatorname{cf}(\kappa)>\boldsymbol{x}_{0}$.

Proof. Define $S_{i}, \rho_{i}, f_{i}$, for $i \in \omega$, inductively. Let $S_{0}=\kappa, \rho_{0}$ the identity. Let $f_{i}$ be thin wrt $S_{i}, \rho_{i}$. Define $S_{i+1}=\bigcup\left\{D_{a}^{f_{i}}: \alpha \in C\right\}$. Then Lemma 1 separates $S_{i}-S_{i+1}$. Using this and $(*)$ we can separate $\bigcup\left\{S_{i}-S_{i+1}: i \in \omega\right\}$. It is sufficient to show $\bigcup\left\{S_{i}-S_{i+1}: i \epsilon \omega\right\}=\kappa$, or equivalently, $\bigcap\left\{s_{i}: i \in \omega\right\}=\varnothing$. Let $\rho_{i+1}^{\prime}$ map $D_{c_{a}}^{f_{i}^{i}}$ one-to-one to $B_{a}$, and let

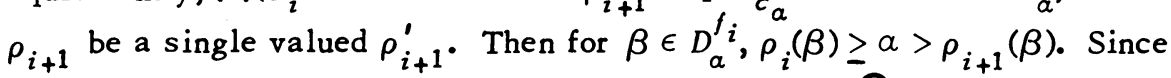
there are no infinite descending sequences of ordinals, $\cap s_{i}=\varnothing$.

This completes the proofs of the induction steps, so the Theorem is proved.

\section{REFERENCES}

1. R. H. Bing, Metrization of topological spaces, Canad. J. Math. 3 (1951), 175-186. MR 13, 264.

2. - A translation of the normal Moore space conjecture, Proc. Amer. Math. Soc. 16 (1965), 612-619. MR 31 \#6201.

3. R. B. Jensen, The fine structure of the constructible hierarchy, Ann. Math. Logic 4 (1972), 229-308; Erratum, ibid. 4 (1972), 443. MR 46 \#834.

4. F. D. Tall, Set theoretic consistency results and topological theorems, Ph.D. Thesis, University of Wisconsin, Madison, Wis., 1969.

5. R. B. Jensen, Souslin's hypothesis is compatible with $\mathrm{CH}, 150 \mathrm{handwritten}$ pages (unpublished).

DEPARTMENT OF MATHEMATICS, UNIVERSITY OF WISCONSIN, MADISON, WISCONSIN 53706

Current address: Department of Mathematics, McGill University, Montreal, Quebec, Canada 